\title{
Effects of Maternal Hypothyroidism on the Pubertal Development in Female Rat Offspring
}

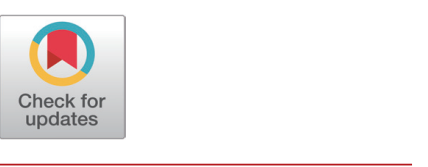

Received: April 3, 2021

Revised: May 6, 2021

Accepted: June 1, 2021

\author{
${ }^{\dagger}$ Corresponding author \\ Sung-Ho Lee \\ Department of Biotechnology, \\ Sangmyung University, Seoul 03016, \\ Korea. \\ Tel: +82-2-2287-5139 \\ Fax: +82-2-2287-0070 \\ E-mail: shlee@smu.ac.kr
}

Copyright $\odot 2021$ The Korean Society of Developmental Biology.

This is an Open Access article distributed under the terms of the Creative Commons Attribution Non-Commercial License (http://creativecommons.org/licenses/ by-nc/4.0/) which permits unrestricted non-commercial use, distribution, and reproduction in any medium, provided the original work is properly cited.

ORCID

Jin-Soo Park

https://orcid.org/0000-0001-9046-2097

Sung-Ho Lee

https://orcid.org/0000-0003-2866-3642

Conflict of interests

The authors declare no potential conflict of interest.

Acknowledgements

This research was funded by a 2020 research Grant from Sangmyung

University (2020-A000-0172)

Authors' contributions

Conceptualization: Lee SH.

Data curation: Lee $\mathrm{SH}$.

Formal analysis: Park JS.

Methodology: Park JS.

Software: Park JS.

Validation: Lee SH, Park JS.

Investigation: Lee $\mathrm{SH}$, Park JS.

Writing-original draft: Lee SH, Park JS.

Writing-review \& editing: Lee SH, Park JS.

\author{
Jin-Soo Park and ${ }^{\dagger}$ Sung-Ho Lee \\ Department of Biotechnology, Sangmyung University, Seoul 03016, Korea
}

\section{Abstract}

The present study was performed to investigate the effect of maternal hypothyroidism and puberty onset in female rat pups. To do this, we employed propylthiouracil (PTU) to prepare a hypothyroid rat model. Pregnant rats were treated with PTU $(0.025 \%)$ in drinking water from gestational day 14 to postnatal day 21 of offspring. Comparison of general indices such as body and tissue weights and puberty indices such as vaginal opening (VO) and tissue histology between control and PTU-treated rats were conducted. There was no significant difference in the date of VO between control and PTU group. The body weights of the PTU group were significantly lower, only $36.8 \%$ of the control group $(p<0.001)$. Although the absolute thyroid weight was not changed by PTU treatment, the relative weight increased significantly about 2.8 times $(p<0.001)$, indicating that hypothyroidism was successfully induced. On the other hand, the absolute weights of the ovary and uterus were markedly decreased by PTU administration $(p<0.001)$, and the relative weight was not significantly changed. The ovarian histology of PTU group revealed the advanced state of differentiation (i.e., presence of corpora lutea). Inversely, the uterine histology of PTU group showed underdeveloped structures compared those in control group. Taken together, the present study demonstrates that our maternal hypothyroidism model resulted in minimal effect on pubertal development symbolized by VO despite of huge retardation in somatic growth. More sophisticatedly designed hypothyroidism model will be helpful to achieve a better understanding of pubertal development and related disorders.

Keywords: Maternal hypothyroidism, Puberty, Ovary, Vaginal opening, Rat

\section{INTRODUCTION}

Thyroid hormones (THs) are vital for the maintenance of normal physiology in humans and animals. Hypothyroidism is a disorder of the endocrine system in which the thyroid does not produce enough THs, affecting up to $5 \%$ of the general population with a further estimated $5 \%$ being undiagnosed in USA (Chiovato et al., 2019). It can cause a wide range of symptoms, such as poor ability to tolerate cold, a feeling of tiredness, constipation, slow heart rate, depression, and weight gain (El-Shafie, 2003; Idris et al., 2005).

In older children and adolescents, the symptoms of hypothyroidism are similar to those in adult, and particularly, include irregular menstrual cycles and delayed puberty in girls (Anasti et al., 1995; 
Ethics approval

The animal protocols were approved by the Animal Care and Use Committees at Sangmyung University (approval number R-2001-1).
Counts and Varma, 2009). In hypothyroid rodents, however, the delay of puberty onset in female is uncertain while it is prominent in male (Wilen et al., 1981; Shibutani et al., 2009). So far, this discrepancy has not been investigated in detail.

6-n-propyl-2-thiouracil (PTU) is a medication used to treat hyperthyroidism, also is widely applied to induce hypothyroidism in experimental rodents (Mandel and Cooper, 2001; Mallela et al., 2014). The present study was performed to understand the relationship between maternal hypothyroidism and puberty onset in female rat offspring. To do this, we employed PTU-induced hypothyroid rat model. Based on our pilot studies, we used slightly modified PTU model (Hapon et al., 2007; Zamoner et al., 2008; Tanaka et al., 2019). Comparison of general indices such as body and tissue weights, and puberty indices such as vaginal opening (VO) and tissue histology between control and PTU-treated rats were conducted.

\section{MATERIAL \& METHODS}

\section{Chemicals and animals}

6-Proryl-2-thiouracil (PTU; CAS no. 51-52-5, purity>99.0\%) was purchased from Tokyo chemical industry (Tokyo, Japan). All other chemicals were purchased from Sigma-Aldrich (St. Louis, MO, USA). Pregnant Sprague-Dawley rats were purchased from DBL (Eumseong, Korea) at gestational day (GD) 12 . The animal rooms were maintained in air-conditioned temperature $22 \pm 1^{\circ} \mathrm{C}$, relative humidity $40 \pm 5 \%$ with 12 -hour light/dark cycle. Dam and offspring were fed ad libitum with Purina Mouse Diets, purchased from Biopia (Gunpo, Korea). The animal protocols were approved by the Animal Care and Use Committees at Sangmyung University (approval number R-2001-1). All the animals received humane care in accordance with the guides for animal experiments of the Association for Assessment and Accreditation of Laboratory Animal Care (AAALAC).

\section{PTU treatment and tissue preparations}

Dams were given drinking water containing $0.025 \%$ of PTU w/v or water alone from GD14 to at postnatal day (PND) 21 as previously reports with slightly modification, for longer exposure period and lower dose than original procedures (Hapon et al., 2007; Zamoner et al., 2008). The start date of PTU treatment was determined to be the 13th day of gestation (GD13) according to the report that thyroid hormone receptors were first expressed in the fetus on the GD14 of pregnancy (Dowling et al., 2000). PTU containing water was freshly changed every two days. Pups of control group were weaned at PND 21, and dams in PTU-exposed group were further maintained for additional 7 days without PTU-treatment for further nursing of offspring showing severe growth suppression due to hypothyroidism (Tanaka et al., 2019). Body weights and the dates of VO were measured until PND 36. Animals were sacrificed, and the tissue weights were measured between 18:00-19:00 pm. The tissues were fixed in paraformaldehyde (4\%) for histological study.

\section{Paraffin section}

Fixed tissues (thyroids, ovaries, uteri and ovarian fat fads) were dehydrated in graded concentrations of ethanol (70\%, 80\%, 90\%, 95\%, and 100\%; Duksan, Korea) for 1 hour 30 minutes in each with gentle shaking and soaked in absolute ethanol overnight. The tissues were immersed in xylene (Samchun Chemical, Seoul, Korea) for 30 minutes, 3 times and in paraffin (Avantik Biogroup, Pine Brook, NJ, USA) at $56^{\circ} \mathrm{C}$ for 30 minutes, 3 times. The tissues were embedded in paraffin and sectioned (Microm, Walldorf, Germany) at $5 \mu \mathrm{m}$. The samples were attached on 
microscope slides (Marienfeld, Lauda-Königshofen, Germany) and the slides were stained with hematoxylin (Sigma-Aldrich) and eosin (Across, Carson, CA, USA) for 5 minutes, respectively. The width of the thyroid follicle lumen was measured using Image-J software, and other histological parameters such as thickness of the follicular cells were measured.

\section{Statistical analysis}

Values were expressed as mean \pm SE Data were analyzed using Student's $t$-test and MannWhitney $\mathrm{U}$ test as indicated. $p<0.05$ was considered statistically significant. Calculations were performed using Graphpad Software Prism version 5.

\section{RESULTS}

\section{Changes in body weights}

Fig. 1 shows that maternal PTU administration significantly inhibited weight gain in hypothyroid pups $(p<0.001)$. The offspring weights of two groups differed significantly from PND7. The gap in average weight continued to increase thereafter until PND 35.

\section{Analyses of vaginal opening}

There was no significant difference in the date of VO, which is a hallmark of the puberty onset, between control and PTU group (Fig. 2). In both groups, VO of the most objects were observed at PND36, but some animals of PTU group had checked relatively later.

\section{The body weights at vaginal opening day}

The average body weight at the time of $\mathrm{VO}$ showed a huge difference between the two groups. The body weights of the PTU group were significantly lower, only $36.8 \%$ of the control group $(p<0.001$, Table 1).

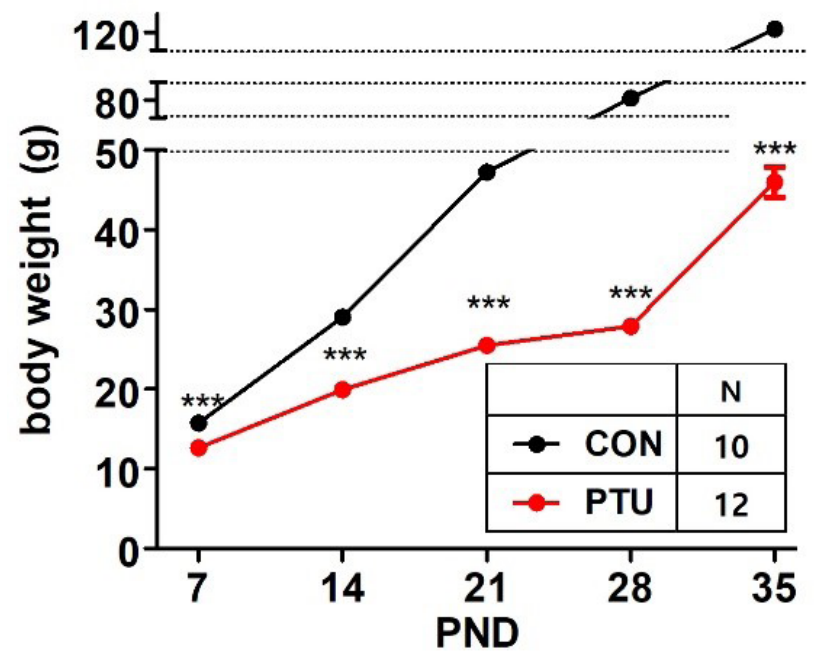

Fig. 1. PTU-induced maternal hypothyroidism inhibits offspring growth until postnatal day (PND) 36. Mothers of PTU group received PTU in drinking water from GD 14 to PND 21 and then Tap water until PND 28. Pups of control group were weaned at PND 21. ${ }^{* \star *} p<0.001$, compared to CON group by Mann Whitney or unpaired $t$-test. PTU, propylthiouracil; PND, postnatal day. 


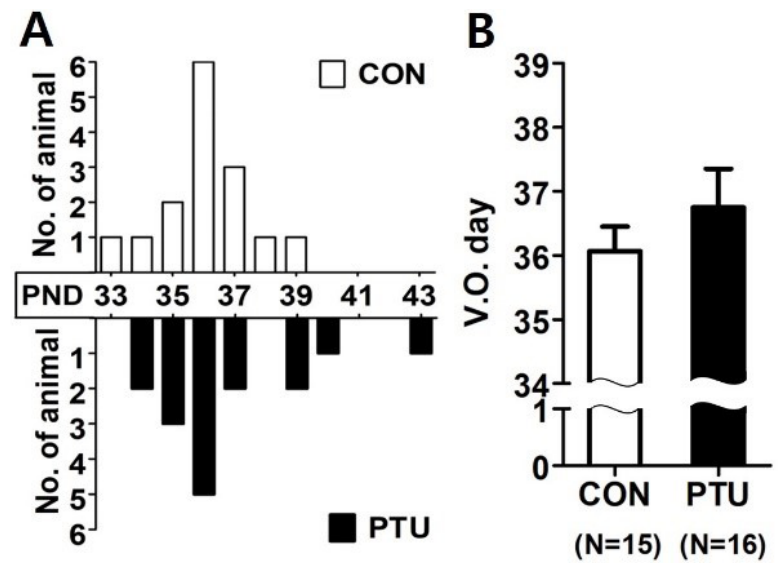

Fig. 2. Effect of maternal hypothyroidism on puberty onset of female offspring. Vaginal opening and smears were examined daily after weaning. (A) Comparison of mean vaginal opening (VO) day between control and PTU-treated groups. (B) Comparison of the daily VO profiles between control and PTUtreated groups. In both groups, VO occurred the most on the PND 36. PTU, propylthiouracil.

Table 1. Effect of maternal PTU exposure during GD 13 through PND 21 on offspring body weight at vaginal opening day

\begin{tabular}{ccc}
\hline \hline & CON & PTU \\
\hline Body weight $(\mathrm{g})$ & $138.0 \pm 3.69$ & $50.8 \pm 3.15^{\text {** }}$ \\
\hline
\end{tabular}

Values are expressed as mean \pm SE. CON $(n=12)$, PTU $(n=13)$.

Differences between groups were considered as significant at $p<0.05$ and analyzed with Mann-Whitney test $\left({ }^{* * *} p<0.001\right)$.

PTU, propylthiouracil.

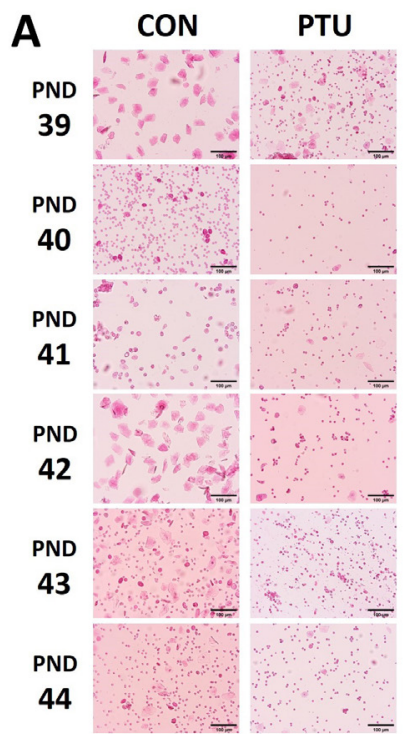

B

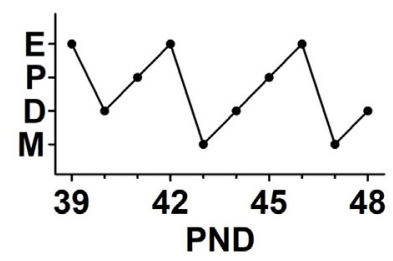

C

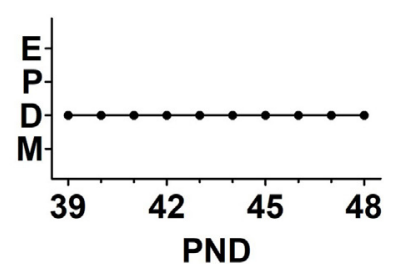

Fig. 3. Daily analysis of estrous cyclicity in CON and PTU rats after VO. (A) Representative patterns of vaginal smears for 6 days post VO from CON (left) and PTU (right) rats. Smears were stained with $0.1 \%$ Eosin solution $($ Bar $=100 \mu \mathrm{m})$. The vaginal smear contains only keratinocytes at estrus (PND39 and 42 of CON group). Metestrus is characterized by the mix of lymphocytes (small-nucleated cells) and keratinocytes in the vaginal fluid (PND43 of CON group). Diestrus is characterized by the presence of lymphocytes in the vaginal fluid (PND 44 of CON group. During the proestrus stage, nucleated epithelial cells are observed in the vaginal smear (PND41 of CON group). On the other hand, vaginal smears collected from PTU rat reveal the prolonged presence of lymphocytes (i.e., diestrus), indicating a complete alteration of the estrous cycle. The bar represents $100 \mu \mathrm{m}$ (B and C). Representative samples of the cyclicity in CON (B) and PTU (C) groups, respectively. PTU, propylthiouracil; PND, postnatal day; E, estrus; P, proestrus; D, diestrus; $M$, metestrus; $\mathrm{VO}$, vaginal opening. 


\section{Analyses of estrous cyclicity}

Fig. 3 demonstrated the estrous cyclicity observed after VO. Each stage of the cycle was determined by the predominant presence of either lymphocytes, nucleated epithelial cells, or keratinocytes (see figure legend for details). Serial microphotographs of the smeared cells from control animals revealed the good cyclicity while no cyclicity was found in the cells from PTU-treated animals (Fig. 3A). The normal estrous cycle has a characteristic periodicity, with an estrus every 4-5 days. The representative patterns of vaginal smears for 10 days post $\mathrm{VO}$ also showed the four stages in control animals, while only one persistent stage was found in PTU-treated animals (Fig. 3B and C, respectively).

\section{Changes in tissue weights}

Table 2 showed the effect of PTU administration on changes in tissue weight at VO day. The absolute weight of all tissues except for the thyroid gland decreased significantly, and the relative weights increased significantly except for the ovary and uterus (Table 2). Although the absolute thyroid weight was not changed by PTU treatment, the relative weight increased significantly about 2.8 times $(p<0.001)$, indicating that the hypothyroidism pursued by this experimental model was successfully induced. On the other hand, the absolute weights of the ovary and uterus were markedly decreased by PTU administration $(p<0.001)$, the relative weights were not significantly changed. This phenomenon is interesting, because it suggests that the pubertal growth of thyroid gland might be proceeded regardless of somatic growth, but not in other organs.

\section{Histological analysis}

At PND 36, when VO was not checked in control while VO was recorded in PTU animal, tissue samplings for histological study were performed. Although the absolute weights of the thyroid gland of both control and PTU group were similar (Table 2), histological analysis revealed that the area of thyroidal follicular lumen and the width of follicular epithelial cells were greatly reduced in the PTU group. As expected, the size of the ovaries was markedly reduced in PTU group (Table 2). There were many primary and secondary follicles and no corpus luteum in the control group, but relatively few primary and secondary follicles and many tertiary follicles and corpus luteum-like structures were found in the PTU group (Fig. 4). The size of the uterus was relatively small in the PTU group (Table 2), however, the endometrium, lumen, and muscle layers of the PTU group were underdeveloped states compared to those of control group. In case of ovarian fat pad, more cell nuclei and reductions in cell area were observed in the PTU group.

Table 2. Effect of maternal PTU administration on tissue weight change

\begin{tabular}{cccccc}
\hline \hline & \multicolumn{2}{c}{ Absolute tissue weight $(\mathrm{mg})$} & & \multicolumn{2}{c}{ Relative tissue weight } \\
\cline { 2 - 3 } \cline { 5 - 6 } & CON & PTU & & CON & PTU \\
\hline Thyroid & $4.5 \pm 0.25$ & $4.7 \pm 0.29$ & & $3.6 \pm 0.22$ & $10.2 \pm 0.73^{* * *}$ \\
Ovary & $20.4 \pm 1.48$ & $7.3 \pm 0.32^{* * *}$ & & $16.4 \pm 1.21$ & $15.4 \pm 0.75$ \\
Uterus & $206.7 \pm 23.22$ & $100.1 \pm 27.85^{*}$ & & $16.4 \pm 1.71$ & $21.9 \pm 6.88$ \\
Adrenal & $15.0 \pm 0.69$ & $8.4 \pm 0.39^{* * *}$ & & $12.0 \pm 0.62$ & $19.1 \pm 0.90^{* * *}$ \\
Kidney & $661.9 \pm 19.07$ & $306.7 \pm 8.26^{\star * *}$ & & $53.0 \pm 1.59$ & $69.5 \pm 1.78^{\star * *}$ \\
Spleen & $466.2 \pm 17.78$ & $250.1 \pm 15.08^{* * *}$ & & $37.3 \pm 1.17$ & $56.5 \pm 2.76^{* \star *}$ \\
\hline
\end{tabular}

Relative tissue weight calculations are as follows, depending on the tissue.

Thyroid, ovary, adrenal: relative tissue weight=[Tissue weight $(\mathrm{mg}) /$ Bodyweight $(\mathrm{g})] \times 100$.

Uterus, kidney, spleen: relative tissue weight=[Tissue weight (mg)/Bodyweight $(\mathrm{g})] \times 10$.

Values are expressed as mean \pm SE Groups marked with an asterisk are significantly different from control group, ${ }^{*} p<0.05$, ${ }^{* *}$ $p<0.01,{ }^{* * *} p<0.001$

PTU, propylthiouracil. 


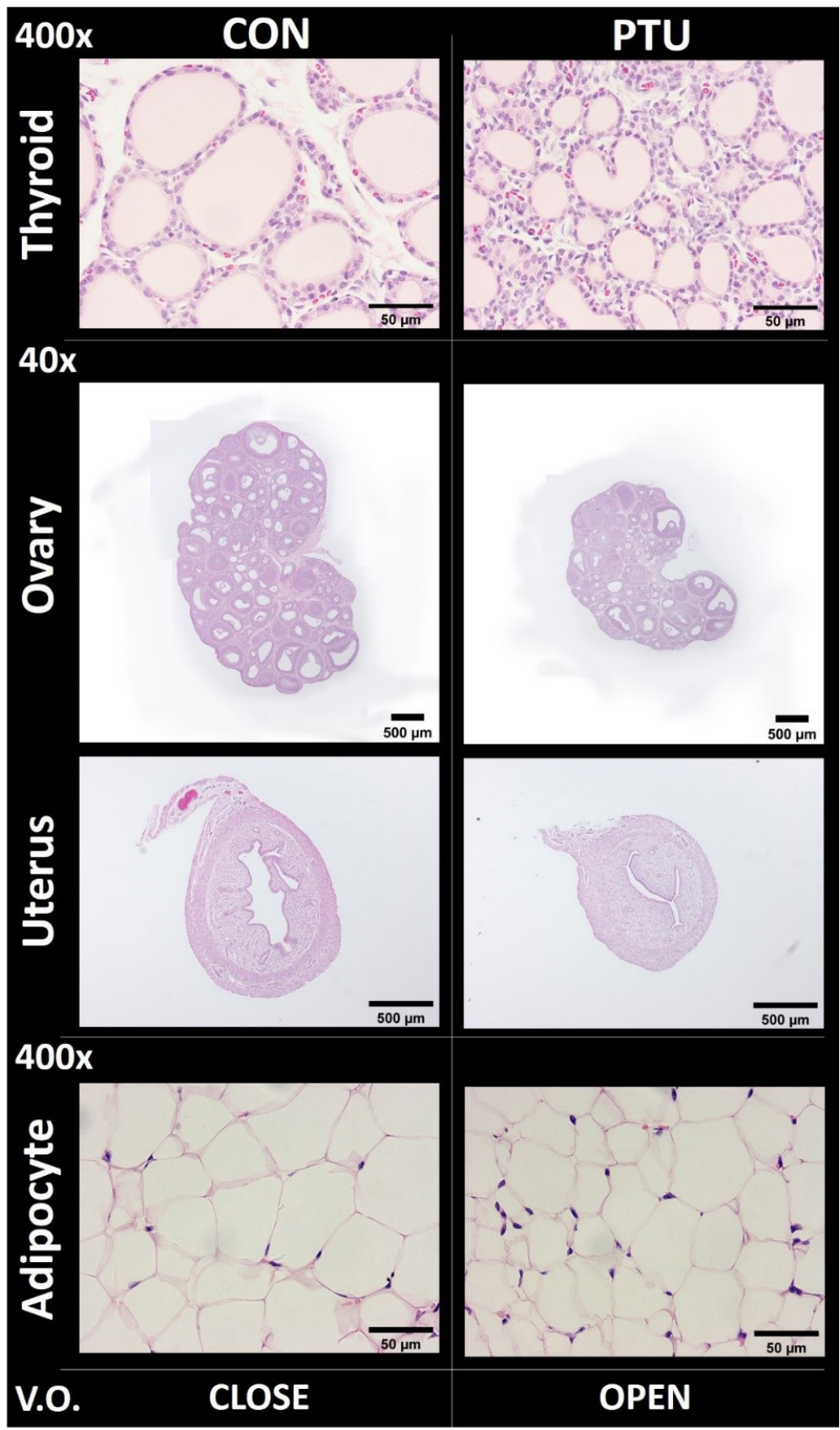

Fig. 4. Histological analyses of the tissues from female offspring exposed to maternal treatment with PTU. At PND36, animals were sacrificed and the thyroids, ovaries, uteri, ovarian fat pads were immediately collected and fixed in $4 \%$ paraformaldehyde. The samples were applied to the standard paraffin section and hematoxylin \& eosin staining method. Representative microphotographs $(\times 40$; ovaries, uterus. $\times 400$; thyroid, ovarian fat pad-adipocytes). PTU, propylthiouracil; PND, postnatal day.

\section{Histological parameter analysis}

Table 3 showed histological parameters such were luminal area, epithelial cell width, gland number, follicle number, and adipocyte cell number. In the thyroid of PTU group, follicle luminal and epithelial width were significantly reduced $(p<0.001)$. In the uterus of PTU group, widths of perimetrium and myometrium, and epithelial area were significantly reduced $(p<0.01$ and $p<0.001$, respectively). In the ovary of PTU group, numbers of primary, secondary and Graafian folicles were 
Table 3. Effects of maternal PTU treatment on histological parameters of thyroid, ovary, uterus and adipocyte at PND36

\begin{tabular}{cccc}
\hline \hline & & CON & PTU \\
\hline Thyroid & Area of follicle lumen $\left(\mu \mathrm{m}^{2}\right)$ & $3,106.60 \pm 130.025$ & $1,366.92 \pm 34.059^{* * *}$ \\
& Width of epithelium $(\mu \mathrm{m})$ & $7.73 \pm 0.166$ & $5.33 \pm 0.103^{* * *}$ \\
\hline Uterus & Width of perimetrium $(\mu \mathrm{m})$ & $75.60 \pm 5.476$ & $53.82 \pm 3.804^{* *}$ \\
& Width of myometrium $(\mu \mathrm{m})$ & $83.04 \pm 4.664$ & $64.22 \pm 3.651^{* *}$ \\
& Number of endometrium gland & $4.67 \pm 0.667$ & $4.67 \pm 1.453$ \\
& Area of epithelium $\left(\mu \mathrm{m}^{2}\right)$ & $29.45 \pm 3.196$ & $12.97 \pm 1.098^{* * *}$ \\
\hline \multirow{2}{*}{ Ovary } & Number of primary follicles & $21.67 \pm 3.383$ & $32.67 \pm 9.333$ \\
& Number of secondary follicles & $24.67 \pm 6.386$ & $37.33 \pm 4.177$ \\
& Number of Graafian follicles & $6.00 \pm 2.517$ & $3.67 \pm 1.333$ \\
& Number of corpus lutea & 0 & $2.33 \pm 2.333$ \\
\hline & Area of adipocyte $\left(\mu \mathrm{m}^{2}\right)$ & $1,861.93 \pm 69.279$ & $1,318.18 \pm 58.123^{* * *}$ \\
\hline
\end{tabular}

Mother rats were supplied with water or $0.025 \%$ propylthiouracil from gestation day 14 to offspring postnatal day (PND) 21 . After PND 21, offspring rats were supplied with non-propylthiouracil water until sacrifice. The parameters were measured by ImageJ software.

Values are shown as mean \pm SE.

Significant differences are marked with asterisks ( ${ }^{*} p<0.05 ;{ }^{* *} p<0.01 ;{ }^{* * *} p<0.001$; compared with the CON group). PTU, propylthiouracil.

not significantly changed, while the number of the corpus luteum was $2.33 \pm 2.333$ in PTU group and 0 in CON group. In the ovarian fat pad adipocytes of PTU group, cell areas were significantly reduced $(p<0.001)$.

\section{DISCUSSION}

The primary function of the thyroid is the production of the iodine containing THs, triiodothyronine (T3) and thyroxine (T4) and the peptide hormone calcitonin (Hall and Guyton, 2011). THs have a wide range of effects on the human body in metabolism, cardiovascular function, development and play a role in maintaining normal sexual function (Colledge et al., 2010). Two main TH-associated thyroid disorders are hyperthyroidism and hypothyroidism; hyperthyroidism is characterized by excessive secretion of THs, and hypothyroidism is characterized by a deficient secretion of THs (Taylor et al., 2018). In the present study, the absolute weight of the thyroid from female offspring was not changed by PTU treatment from GD14 through PND 21. However, the relative weight was increased significantly about 2.8 times $(p<0.001)$, indicating that the hypothyroidism pursued by this experimental model was successfully induced.

Pituitary TSH, hypothalamic TRH, and thyroid THs form the hypothalamic-pituitary-thyroid hormonal axis (HPT), and in general, elevated blood levels of THs inhibit the release of TRH and TSH, whereas the opposite effect occurs when serum TH levels decrease (Choksi et al., 2003). Furthermore, HPT and the hypothalamic-pituitary-gonadal axis (HPG) are physiologically related and act together as a unified system in some pathological conditions (Doufas and Mastorakos, 2000). There are several reports that speculate how thyroid disorders induce abnormal puberty based on interaction between the two neuroendocrine axes (Castañeda Cortés et al., 2014; Fekete and Lechan, 2014; Kiyohara et al., 2017 ). The junction of the two axes is estimated to be hypothalamic gonadotropin-inhibitory hormone $(\mathrm{GnIH}$, also known as $\mathrm{RF}$ amide-related peptide coded by the NPVF gene); TH-mediated HPG regulation may be initiated by decrease of GnIH expression, 
which acts at the most upstream level of the HPG axis by inhibiting the activity of GnRH neurons to reduce circulating levels of gonadotropins (LH and FSH) and gonadal sex steroids (Tsutsui et al., 2018). The precise mechanism of TH action, however, on puberty onset is still unclear. In addition, there is conflicting results regarding the effect of abnormal thyroid status on reproductive development. For example, hypothyroidism in girls can cause alterations in the pubertal process, usually a delay, but occasionally it can result in pseudo-precocious puberty (Doufas and Mastorakos, 2000). In the present study, we found that there was no significant difference in the date of VO between control and PTU group. It is intriguing that, the body weight and ovarian weight of PTU group were greatly reduced compared to the weights of control group while VO could be checked almost simultaneously in both groups. The ovary of PTU group might have equivalent ability to induce VO, despite of the tiny size. At this time, we tentatively concluded that there is no strong correlation between body/ovarian weights and $\mathrm{VO}$ when maternal hypothyroidism model is employed. To confirm this idea, one should carefully compare the available data from several precocious and/ or delayed puberty of rat models (Lee et al., 2009; Sun et al., 2021).

Regarding the determination of the puberty onset in female rodents, date of VO has been accepted as most reliable phenotypic signal. We often found that incomplete opening of vagina still collection of epithelial cells is possible particularly in PTU group, and these cases could make a serious error. Therefore, some auxiliary index will be helpful, and serum hormone levels and histology might be served as final confirming index at postmortem.

Taken together, the present study demonstrates the maternal hypothyroidism induced by PTU administration exerts minimal effect on pubertal development symbolized by VO despite of huge retardation in somatic growth. Further studies using more sophisticatedly designed hypothyroidism model will be especially important, and it will help to advance our understanding of pubertal development and related disorders where and when HPT and HPG axis are mutually controlled.

\section{REFERENCES}

Anasti JN, Flack MR, Froehlich J, Nelson LM, Nisula BC (1995) A potential novel mechanism for precocious puberty in juvenile hypothyroidism.J Clin Endocrinol Metab 80:276-279.

Castañeda Cortés DL, Langlois VS, Fernandino JI (2014) Crossover of the hypothalamic pituitaryadrenal/interrenal, -thyroid, and -gonadal axes in testicular development. Front Endocrinol 5:139.

Chiovato L, Magri F, Carlé A (2019) Hypothyroidism in context: Where we've been and where we're going. Adv Ther 36:47-58.

Choksi NY, Jahnke GD, St Hilaire C, Shelby M (2003) Role of thyroid hormones in human and laboratory animal reproductive health. Birth Defects Res B: Dev Reprod Toxicol 68:479-491.

Colledge NR, Walker BR, Ralston SH (2010) Davidson's Principles and Practice of Medicine. 21st ed. Elsevier, Edinburgh, UK.

Counts D, Varma SK (2009) Hypothyroidism in children. Pediatr Rev 30:251-258.

Doufas AG, Mastorakos G (2000) The hypothalamic-pituitary-thyroid axis and the female reproductive system. Ann NY Acad Sci 900:65-76.

Dowling ALS, Martz GU, Leonard JL, Zoeller RT (2000) Acute changes in maternal thyroid hormone induce rapid and transient changes in gene expression in fetal rat brain. J Neurosci 20:2255-2265.

El-Shafie KT (2003) Clinical presentation of hypothyroidism. J Family Community Med 10:5558. 
Fekete C, Lechan RM (2014) Central regulation of hypothalamic-pituitary-thyroid axis under physiological and pathophysiological conditions. Endocr Rev 35:159-194.

Hall JE, Guyton A (2011) Guyton and Hall Textbook of Medical Physiology. 12th ed. Elsevier, Philadelphia, PA.

Hapon MB, Varas SM, Giménez MS, Jahn GA (2007) Reduction of mammary and liver lipogenesis and alteration of milk composition during lactation in rats by hypothyroidism. Thyroid 17:11-18.

Idris I, Srinivasan R, Simm A, Page RC (2005) Maternal hypothyroidism in early and late gestation: Effects on neonatal and obstetric outcome. Clin Endocrinol 63:560-565.

Kiyohara M, Son YL, Tsutsui K (2017) Involvement of gonadotropin-inhibitory hormone in pubertal disorders induced by thyroid status. Sci Rep 7:1042.

Lee SY, Jang YS, Lee YH, Seo HH, Noh KH, Lee SH (2009) Advanced onset of puberty in high-fat diet-fed immature female rats: Activation of KiSS-1 and GnRH expression in the hypothalamus. Dev Reprod 13:183-190.

Mallela MK, Strobl M, Poulsen RR, Wendler CC, Booth CJ, Rivkees SA (2014) Evaluation of developmental toxicity of propylthiouracil and methimazole. Birth Defects Res B Dev Reprod Toxicol 101:300-307.

Mandel SJ, Cooper DS (2001) The use of antithyroid drugs in pregnancy and lactation. J Clin Endocrinol Metab 86:2354-2359.

Shibutani M, Woo GH, Fujimoto H, Saegusa Y, Takahashi M, Inoue K, Hirose M, Nishikawa A (2009) Assessment of developmental effects of hypothyroidism in rats from in utero and lactation exposure to anti-thyroid agents. Reprod Toxicol 28:297-307.

Sun J, Shen X, Liu H, Lu S, Peng J, Kuang H (2021) Caloric restriction in female reproduction: Is it beneficial or detrimental? Reprod Biol Endocrinol 19:1.

Tanaka T, Masubuchi Y, Okada R, Nakajima K, Nakamura K, Masuda S, Nakahara J, Maronpot R, Yoshida T, Koyanagi M, Hayashi SM, Shibutani M (2019) Ameliorating effect of postweaning exposure to antioxidant on disruption of hippocampal neurogenesis induced by developmental hypothyroidism in rats. J Toxicol Sci 44:357-372.

Taylor PN, Albrecht D, Scholz A, Gutierrez-Buey G, Lazarus JH, Dayan CM, Okosieme OE (2018) Global epidemiology of hyperthyroidism and hypothyroidism. Nat Rev Endocrinol 14:301-316.

Tsutsui K, Son YN, Kiyohara M, Miyata I (2018) Discovery of GnIH and its role in hypothyroidism-induced delayed puberty. Endocrinology 159:62-68.

Wilen R, Bastomsky CH, Naftolin F (1981) Control of puberty in female rats: The effect of PTUinduced hypothyroidism and systematic undernutrition. Pediatr Res 15:169-171.

Zamoner A, Barreto KP, Filho DW, Sell F, Woehl VM, Guma FC, Pessoa-Pureur FR, Silva F (2008) Propylthiouracil-induced congenital hypothyroidism upregulates vimentin phosphorylation and depletes antioxidant defenses in immature rat testis.J Mol Endocrinol 40:125-135. 